\title{
Determining Six Cardiac Conductivities from Realistically Large Datasets
}

\author{
Barbara M. Johnston ${ }^{\mathrm{a}, *}$, Peter R. Johnston ${ }^{\mathrm{a}}$ \\ ${ }^{a}$ School of Natural Sciences and Queensland Micro and Nanotechnology Centre \\ Griffith University \\ Nathan \\ Queensland \\ Australia, 4111 \\ Phone: 61-7-3735-4405 \\ Fax: $61-7-3735-7656$
}

\begin{abstract}
Simulation studies of cardiac electrophysiological behaviour that use the bidomain model require accurate values for the bidomain extracellular and intracellular conductivities to produce useful results. This work considers an inversion algorithm, which has previously been shown, using simulated data, to be capable of retrieving six bidomain conductivities and the fibre rotation angle from measurements of electric potential made in the heart. The aim here is to see whether it is possible to improve the accuracy of the retrieved parameters. The scenario of retrieving only conductivities and not fibre rotation is examined but this does not lead to a worthwhile improvement in retrieval accuracy. It is also found that it is possible to retrieve the bidomain conductivities using not two but just one pass of the algorithm, made on a 'widely-spaced' electrode set. This appears to work because the algorithm is still very sensitive to the extracellular conductivities. However, the single-pass method is not recommended because the intracellular conductivities that are retrieved are not as accurate as those that are retrieved in the usual two-pass method, particularly for higher values of added noise. The second part of this work considers retrieving the six conductivities and fibre rotation from realistically large sets of potential measurements and identifies the best data analysis method. It is found that, even with added noise of up to $40 \%$, the extracellular conductivities can still be retrieved extremely accurately (relative errors of around $2 \%$ on average) and so can
\end{abstract}

\footnotetext{
*Corresponding author

Email address: Barbara.Johnston@griffith.edu.au (Barbara M. Johnston)
} 
the intracellular longitudinal conductivities and fibre rotation (errors less than $8 \%$ on average). The remaining intracellular conductivities have errors that are generally less than twice the added noise, particularly for the higher noise values.

Keywords: bidomain model, cardiac conductivities, electrode array, inverse methods, simulation, electrophysiology

\section{Introduction}

Increases in computational power over the past ten years or so have led to major advances in the ability of researchers to realistically simulate cardiac electrophysiological behaviour and even to work towards the use of heart models that can guide clinical decisions. Along with these advances, has come the realisation of the importance of accurate values for the parameters in these models. For example, this is the case for the conductivity values used in the bidomain model that is commonly used to model cardiac tissue $[1,2]$. In addition, not only is it essential to find conductivity values that represent normal tissue, it is also necessary to understand how these are affected in diseased and damaged tissue $[1,3]$.

Despite the bidomain model being used for modelling electrophysiological phenomena for nearly fifty years [1], only three sets of experimentally determined bidomain conductivities exist $[4,5,6]$. Unfortunately, these values are inconsistent [7] and can lead to very different results in simulation studies [8]. Moreover, these studies assume that the conductivities in the directions transverse to the cardiac fibres (that is within the sheet of fibres and between the sheets of fibres) are equal [2]. This assumption is inconsistent with the results of imaging studies of ventricular architecture [2] and has also been shown to be invalid by experimental studies that have demonstrated that there are three distinct propagation directions in cardiac ventricular tissue $[9,10]$. The only two available datasets that do not make this assumption $[11,12]$ have not been fully experimentally determined and also produce inconsistent results in simulations [13].

There are two areas of major challenge associated with determining accurate conductivity values. One is associated with the practicalities of actually making the measurements and the other is related to the computational difficulties that occur because the problem of retrieving the conductivity values from measurements of potential is mathematically ill-posed.

Recent work in the first of these areas relates to the design and fabrication of micro-electrodes that can be used to make measurements that 
will lead to values for cardiac conductivities. For example, Hooks and Trew [10] have constructed a plunge electrode array, which was used to measure monodomain conductivities. This work was extended [14] to a 325 electrode array that was used to demonstrate the electrically orthotropic nature of cardiac ventricular tissue. Other groups $[15,16]$ are working on an approach that uses MEMS fabricated blocks in conjunction with multi-site interstitial stimulation.

Some recent work in the second area includes mapping the electrical activation of the tissue and then using a least squares and singular value decomposition method to obtain the conductivities [17], while another approach [18] looks at breaking the problem into a subset of computationally tractable sub-problems. One recent promising approach that could be used to overcome the difficulties associated with the inverse problem of estimating parameters from non-invasive measurements is the Reduced-order Unscented Kalman Filter method [19], which has been used successfully, using simulated measurements, to identify material parameters in a non-linear mechanical model of the left ventricle.

Proof of concept has recently been shown in silico by the present authors $[20,21]$ for a technique that can determine the six bidomain conductivity values (two domains with three propagation directions in each) that are required to fully describe cardiac tissue conductivity [22, 9]. This is achieved by applying a sub-threshold stimulating current during the nonexcitory phase of the cardiac cycle (ST segment) and making measurements, using a multi-electrode array, in vivo in cardiac ventricular tissue in an animal model. These measurements are then used in conjunction with a novel mathematical inversion method to retrieve the conductivities. An additional parameter, the fibre rotation angle, is also retrieved with this method. This is the angle through which the sheets of cardiac fibres rotate relative to one another between the inner and outer heart surfaces. A recent implementation of the inversion routine on GPUs [23] has resulted in a speedup that has allowed a more realistic investigation into the accuracy of these retrievals to be undertaken.

The purpose of this work is firstly to examine aspects of the inversion method to see if it is possible to improve the accuracy of the retrieved conductivities, in particular the intracellular conductivities, which to date have not been retrieved nearly as accurately as the extracellular conductivities. The second and major aspect of this work is to consider a realistic simulation where large numbers of potential measurements would be recorded on the measuring array and to examine the accuracy of the inversion technique.

This paper presents the model and solution technique in Section 2 and 
the inversion algorithm is discussed in Section 3. Various aspects of the inversion algorithm, such as the effect of retrieving the fibre rotation in addition to the conductivities, and the effect of using a single pass of the algorithm rather than two passes, are examined in the first part of Section 4. The second part considers sets of one hundred measurements of potential that are used to mimic a realistic experimental scenario and from these one hundred sets of parameters are determined for a range of noise levels associated with two starting sets of six conductivity values $[11,12]$. Various data analysis techniques are considered for dealing with the non-physiological values that are sometimes found, while results for the 'best' technique are presented along with conclusions in the final section.

\section{Model and Solution Method}

\subsection{Model Geometry}

Cardiac tissue consists of parallel strands of cells, arranged in sheets that rotate relative to one another along a line between the epicardium (outer heart surface) and the endocardium (inner heart surface). It is well-known that current is able to flow more easily in the longitudinal $(l)$ direction along the fibres, than transverse $(t)$ to the fibres within the sheet or between, that is normal $(n)$ to, the sheets of fibres.

The model considered here is a block of cardiac ventricular tissue with dimensions $2 \mathrm{~cm} \times 2 \mathrm{~cm} \times 1 \mathrm{~cm}$ in $(x, y, z)$ space, lying between the $x-y$ plane at $z=0$, which represents the epicardium, and the $x-y$ plane at $z=1$, which represents the endocardium. From $z=1$, extending to infinity in the positive $z$ direction there is assumed to be a volume of blood that is in contact with the endocardium. The ventricular tissue is approximated using the bidomain model $[24,25,26,1]$. This regards the tissue as consisting of extracellular $(e)$ and intracellular $(i)$ interpenetrating domains, over which the cardiac properties are averaged, thus leading to six bidomain cardiac conductivity values $g_{e l}, g_{e t}, g_{e n}, g_{i l}, g_{i t}, g_{i n}$ being required in the model.

\subsection{Governing Equations and Boundary Conditions}

The potentials in cardiac tissue are given by the bidomain governing equations [25]

$$
\nabla \cdot \mathbf{M}_{i} \nabla \phi_{i}=\frac{\beta}{R}\left(\phi_{i}-\phi_{e}\right) \quad \text { and } \quad \nabla \cdot \mathbf{M}_{e} \nabla \phi_{e}=-\frac{\beta}{R}\left(\phi_{i}-\phi_{e}\right)-I_{s}
$$

where $i=$ intracellular, $e=$ extracellular, $I_{s}$ is a sub-threshold external current source per unit volume applied in the extracellular space, $\phi_{j}(j=i, e)$ is 
the potential, $\beta$ is the surface to volume ratio of the cells and $R$ is the specific membrane resistance. The tensors $\mathbf{M}_{j}(j=i, e)$ take into account the anisotropic nature of the tissue, which comes not only from the varying conductivity values, but also from the fibre rotation within the cardiac tissue [27]. Hence, for a rectangular block of tissue, the $\mathbf{M}_{j}$ are of the form

$$
\mathbf{M}_{j}(x, y, z)=\left(\begin{array}{ccc}
\left(g_{j l}-g_{j t}\right) c^{2}+g_{j t} & \left(g_{j l}-g_{j t}\right) c s & 0 \\
\left(g_{j l}-g_{j t}\right) c s & \left(g_{j l}-g_{j t}\right) s^{2}+g_{j t} & 0 \\
0 & 0 & g_{j n}
\end{array}\right)
$$

where $j=i, e, c=\cos \alpha z$ and $s=\sin \alpha z$ and $\alpha$ is the fibre rotation angle.

The potential distribution in the blood, $\phi_{b}$, is governed by Laplace's equation

$$
\nabla^{2} \phi_{b}=0
$$

The boundary conditions used to solve the model follow from the assumptions that the epicardium is insulated, there is continuity of potential and current at the interface between the tissue and the blood, and the intracellular space is insulated by the extracellular space,

$$
\frac{\partial \phi_{e}}{\partial z}=\frac{\partial \phi_{i}}{\partial z}=0 \quad \text { at } z=0
$$

and

$$
\phi_{e}=\phi_{b}, \quad g_{b} \frac{\partial \phi_{b}}{\partial z}=g_{e n} \frac{\partial \phi_{e}}{\partial z}, \quad \frac{\partial \phi_{i}}{\partial z}=0 \quad \text { at } z=1
$$

where $g_{b}$ is the conductivity of blood and $\phi_{b}$ is the potential in the blood. Also, $\phi_{b} \rightarrow 0$ as $z \rightarrow \infty$, since it is assumed that the blood mass is infinite in the positive $z$ direction. Assuming that the boundaries of the domain are insulated, gives the final boundary conditions at the $x$ and $y$ boundaries

$$
\mathbf{M}_{e} \nabla \phi_{e} \cdot \mathbf{n}=0, \mathbf{M}_{i} \nabla \phi_{i} \cdot \mathbf{n}=0 \quad \text { and } \quad \nabla \phi_{b} \cdot \mathbf{n}=0 .
$$

where $\mathbf{n}$ is the outward pointing normal from the boundary.

The model, equations (1)-(3), subject to boundary conditions (4)-(6), is solved by expanding each of the potentials $\phi_{e}$ and $\phi_{i}$ as a Fourier series, where for $j=i, e$

$$
\begin{aligned}
\phi_{j}(x, y, z) & =\sum_{r=0}^{\infty} \sum_{s=0}^{\infty} C_{r s}^{j}(z) \cos (s \pi y) \cos (r \pi x)+D_{r s}^{j}(z) \sin (s \pi y) \cos (r \pi x) \\
& +E_{r s}^{j}(z) \cos (s \pi y) \sin (r \pi x)+F_{r s}^{j}(z) \sin (s \pi y) \sin (r \pi x)
\end{aligned}
$$


Table 1: Conductivity data (in $\mathrm{mS} / \mathrm{cm}$ ) from the indicated studies.

\begin{tabular}{c|cccccc}
\hline Study & $g_{e l}$ & $g_{\text {et }}$ & $g_{\text {en }}$ & $g_{i l}$ & $g_{i t}$ & $g_{\text {in }}$ \\
\hline Hooks [11] & 2.63 & 2.45 & 1.087 & 2.63 & 0.263 & 0.08 \\
MacLachlan et al. [12] & 2.0 & 1.65 & 1.35 & 3.0 & 1.0 & 0.35 \\
\hline
\end{tabular}

Table 2: Conductivity ratios and space constants (in $\mathrm{mm}$ ) from the indicated studies.

\begin{tabular}{c|ccc|ccc}
\hline Study & $g_{i l} / g_{\text {el }}$ & $g_{i t} / g_{\text {et }}$ & $g_{i n} / g_{\text {en }}$ & $\lambda_{l}$ & $\lambda_{t}$ & $\lambda_{n}$ \\
\hline Hooks [11] & 1.0 & 0.11 & 0.07 & 0.77 & 0.33 & 0.18 \\
MacLachlan et al. [12] & 1.5 & 0.61 & 0.23 & 0.74 & 0.53 & 0.36 \\
\hline
\end{tabular}

and then substituting these into equation (1). This gives two sets of four ordinary differential equations in the coefficients $X_{r s}^{j}(z)$, with $X=C, D, E, F$. A one-dimensional finite difference scheme is set up in the $z$-direction and the above coefficients are found by solving a banded system of linear algebraic equations. Full details of the above method can be found in previous papers $[20,28]$.

\subsection{Model Parameters}

Two datasets of six conductivity values are used in solving the model, that of Hooks [11] and that of MacLachlan et al. [12] (Table 1). Anisotropy ratios $q_{i q} / g_{e q}, q=l, t, n$, for these datasets, are given in Table 2 , as well as

space constants, $\lambda_{q}=\sqrt{\frac{R}{\beta}\left(\frac{g_{i q} g_{e q}}{g_{i q}+g_{e q}}\right)}, q=l, t, n$. In Table 2 , it can be seen that there are large differences in the anisotropy ratios in the various directions, for either conductivity dataset.

As in previous studies [29, 28, 20], it will be assumed that the rotation of the main fibre axis between the epicardium and the endocardium varies linearly with depth through an angle $\alpha$, which is set at $\frac{2 \pi}{3}[29,30]$. Also, to be consistent with earlier work the values of other parameters are taken to be $\beta=2000 \mathrm{~cm}^{-1}$ [31], $R=9100 \Omega \mathrm{cm}^{2}$ [32], $I_{S}=50 \mathrm{~mA} \mathrm{~cm}{ }^{-3}$ [33] and $g_{b}=6.7 \mathrm{mS} / \mathrm{cm}[34]$. This leads to space constants that are in the range 0.18 mm - 0.77 mm (Table 2).

\section{Inversion Algorithm}

\subsection{Tikhonov Regularisation}

The potentials in the forward model (equations (1)-(3)) depend on the required parameters in a nonlinear fashion,

$$
\mathbf{G}(\mathbf{m})=\boldsymbol{\Phi}
$$


where $\mathbf{G}$ represents the model, $\boldsymbol{\Phi}$ is the vector of electrode potentials and $\mathbf{m}=\left[g_{i l}, g_{i t}, g_{i n}, g_{e l}, g_{e t}, g_{e n}, \alpha\right]^{T}$. Since there will be noise in the measurement vector $\boldsymbol{\Phi}$ under experimental conditions, it is necessary to minimise the following Tikhonov functional [35] to obtain an approximation to $\mathbf{m}$ from equation (8)

$$
\|\mathbf{G}(\mathbf{m})-\mathbf{\Phi}\|_{2}^{2}+\gamma^{2}\|\mathbf{m}\|_{2}^{2}
$$

Here $\gamma$ is the regularisation parameter.

Minimisation of the functionals is performed using the SolvOpt solver [36], which minimises non-linear multivariate functions using a modified Shor's $r$-algorithm, which handles constraints using the method of exact penalisation [37]. Modifications that have been made to the method in the SolvOpt algorithm include a technique for choosing the initial step size and the inclusion of an efficient stopping criterion [36]. The constraints applied here for the minimisation are that $0 \leq \alpha \leq \pi$ and that the conductivities are positive. The termination criteria used [36] is that the relative error in the functional is less than $10^{-6}$ for two successive iterations.

The parameters $p_{c}$, found during the inversion, are compared with the generating parameters $p$ from Section 2.3, via the percentage relative error

$$
\text { Percentage relative error }(\%)=\left|\frac{p-p_{c}}{p}\right| \times 100
$$

\subsection{Electrode array}

A $2 \mathrm{~mm} \times 2 \mathrm{~mm} \times 2 \mathrm{~mm}$ multi-electrode array (Figure 1 ) is used to apply a sub-threshold stimulating current and then to make the measurements of potential that are used in the inversion algorithm to retrieve the conductivities and fibre rotation. The array consists of 25 micro-needles each containing three electrodes arranged in three planes, where the spacing between the needles and between the electrodes on the needles is $0.5 \mathrm{~mm}$.

The dimensions of the array are chosen to be consistent with the fourelectrode method of Plonsey and Barr [31], in which two sets of measurements are made using four collinear electrodes, where the outer pair are the source and sink electrodes and the inner pair are the measuring electrodes.

In this array (Figure 1) a 'closely-spaced' (less than the space constant (Table 2) subset of the electrodes is used to make the first set of measurements (the 'first pass') and these are the electrodes on the nine needles that make up the inner square of 25 measuring electrodes (green) and two current electrodes (red, denoted 'S'). The second set of measurements (the 'second 


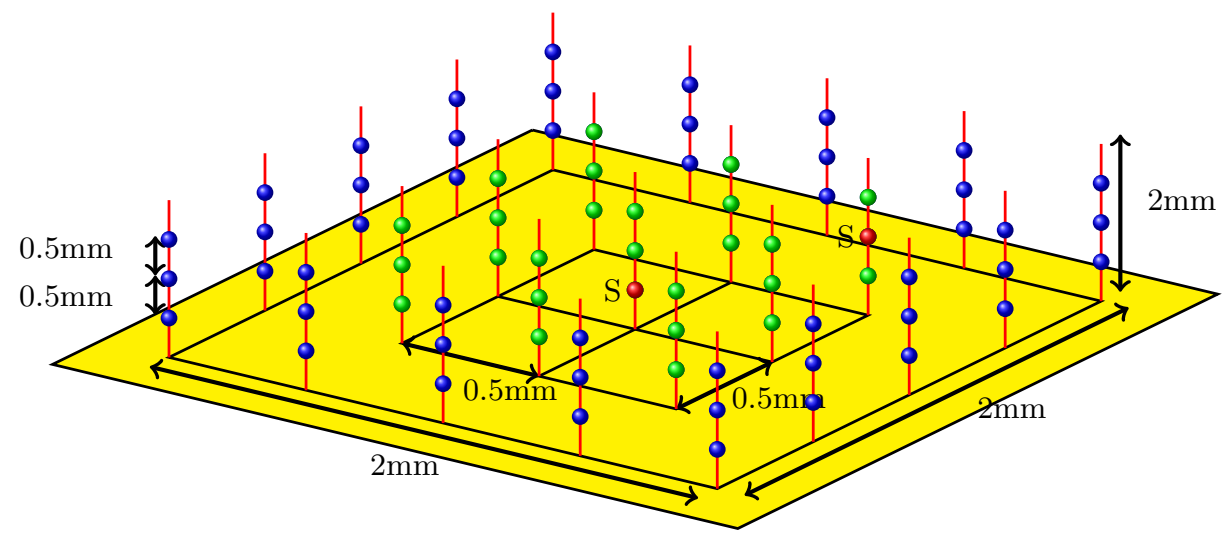

(a) First pass

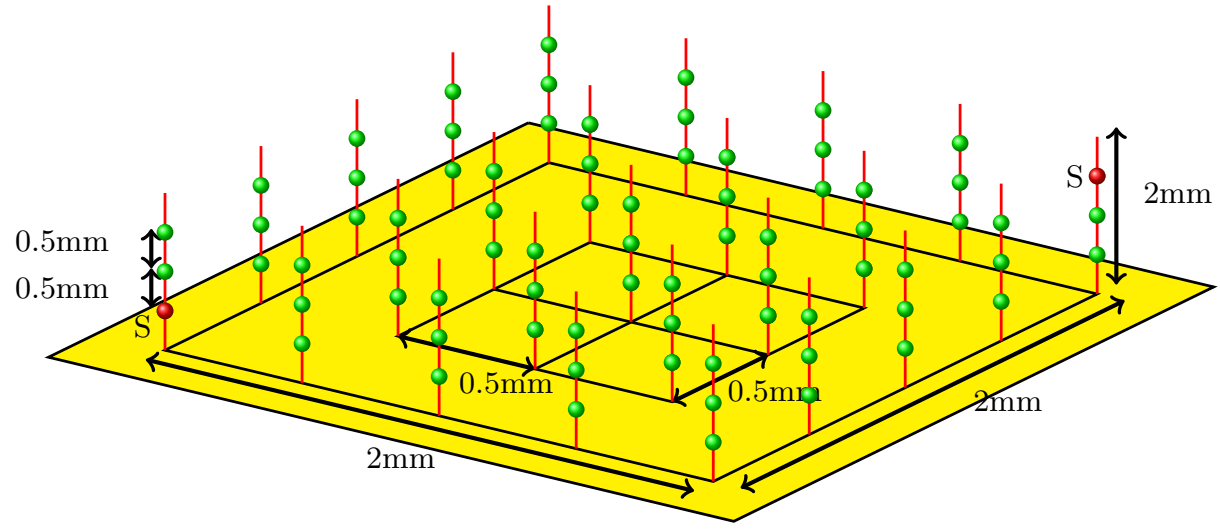

(b) Second pass

Figure 1: The $2 \mathrm{~mm} \times 2 \mathrm{~mm} \times 2 \mathrm{~mm}$ multi-electrode array, comprised of three electrodes per needle in three 'layers', that is used for making the potential measurements. Source and sink electrodes are red and are marked with an ' $\mathrm{S}$ '. The spacing between the electrodes and also between the needles is $0.5 \mathrm{~mm}$. (a) The 'closely-spaced' electrodes on the needles in the inner square (green and red) are used in the first pass. (b) The second pass uses all the electrodes. 
pass') is made using a 'widely-spaced' set (much greater than the space constant), consisting of all 75 electrodes, where the source and sink electrodes (red, denoted ' $\mathrm{S}$ ') are diagonally opposite one another and as far apart as possible. The 'close' spacings correspond to the scenario where almost all the current is in the extracellular space, whereas in the 'wide' spacings some of the current is re-directed into the intracellular space [31].

\subsection{First Pass of the Inversion Algorithm}

The bidomain conductivity values of either Hooks [11] or MacLachlan et al. [12] (Table 1), together with the parameters given in Section 2.3, are used to produce a set of simulated potential measurements on the 'closelyspaced' subset of electrodes in the array (Figure 1). Noise is added to these measurements to produce 'noisy potentials' and the inversion process described in Section 3.1 is then used to retrieve $g_{e l}, g_{e t}, g_{e n}, g_{i l}, g_{i t}, g_{i n}$ and $\alpha$ simultaneously during the first pass through the solver. Initial values for all the conductivities are taken to be $1 \times 10^{-3} \mathrm{~S} / \mathrm{cm}$, except for $g_{\text {in }}=1 \times 10^{-4}$ $\mathrm{S} / \mathrm{cm}$, as it is generally accepted to be an order of magnitude smaller. The initial value for $\alpha$ is taken to be 1 . Testing has indicated that the algorithm is quite insensitive to the choice of the initial values in the first pass.

In the first pass, the Tikhonov functional, equation (9), to be minimised is

$$
f_{1}=\sum_{j=1}^{25}\left[\phi_{M}(j)-\phi_{C}(j)\right]^{2}+\gamma_{1}^{2}\left[g_{i l}^{2}+g_{i t}^{2}+g_{i n}^{2}+g_{e l}^{2}+g_{e t}^{2}+g_{e n}^{2}\right]+\gamma_{2}^{2} \alpha^{2}
$$

where $\phi_{R}(j), R=M, C$ is the difference in potential between electrode $j$ and the reference electrode, as measured $M$ or calculated $C$ by the algorithm. Here $\mathbf{m}=\left[g_{i l}, g_{i t}, g_{i n}, g_{e l}, g_{e t}, g_{e n}, \alpha\right]^{T}$ and the two regularisation parameters $\gamma_{1}$ and $\gamma_{2}$ are taken to be $10^{-2}$ and $10^{-5}$, respectively, when the conductivity units are $\mathrm{S} / \mathrm{cm}$. These were chosen in previous work $[30,20]$ to allow for the different magnitudes of the conductivity values and $\alpha$ and a study into the sensitivity of the algorithm to the choice of the $\gamma_{i}$ values has shown that varying the regularisation parameters by two orders of magnitude does not affect the results obtained.

Sets of noisy potentials are produced for each noise level and from these sets of parameters are retrieved by the inversion algorithm. The means of these parameters are then used in the second pass of the algorithm. As in previous work [21], a set of parameters, where three or more of the values retrieved are outside one standard deviation from the mean, is discarded. In this work this will be referred to as the 'old' method, as a 'new' data analysis method will also be considered. 


\subsection{Second Pass of the Inversion Algorithm}

In the second pass of the inversion algorithm, a similar set of simulated potentials is produced on the 'widely-spaced' electrode set (Figure 1) and then noise is added before the inversion takes place. However, in the second pass, the mean values for $g_{e l}, g_{e t}$ and $g_{e n}$ found in the first pass are held constant. Only values for $g_{i l}, g_{i t}, g_{i n}$ and $\alpha$ are retrieved, using starting values which are their means from the first pass. Therefore, the Tikhonov functional to be minimised is now

$$
f_{2}=\sum_{j=1}^{73}\left[\phi_{M}(j)-\phi_{C}(j)\right]^{2}+\gamma_{1}^{2}\left[g_{i l}^{2}+g_{i t}^{2}+g_{i n}^{2}\right]+\gamma_{2}^{2} \alpha^{2}
$$

As in the first pass, sets of parameters are retrieved and averaged for each noise level and from these percentage relative errors for the parameters are calculated.

\section{Results and Discussion}

\subsection{Alternative inversion approaches}

The goal of the first part of this work is to study two aspects of the inversion algorithm to see if it is possible to retrieve the desired parameters (six conductivities and fibre rotation) more accurately, and, in particular the intracellular conductivity values.

\subsubsection{A single-pass inversion method}

Here we investigate whether it is possible to retrieve the parameters in just one pass. Obviously, we would need to use a 'widely-spaced' electrode set, since it must be capable of retrieving both the intra- and extracellular conductivities, and we already know, from previous work [20], that a 'closely-spaced' electrode set cannot achieve this.

A study was performed using the usual second-pass electrode array (Figure 1) and three sets of 20 simulated measurements of potential were produced by adding noise of 1,2 and $5 \%$ to the potentials on the electrodes. The usual first pass initial values (Section 3.3) were used. The inversion algorithm was then applied to find all 7 parameters $\left(g_{e l}, g_{e t}, g_{e n}, g_{i l}, g_{i t}, g_{i n}\right.$ and $\alpha$ ) at once. The percentage relative errors for the three extracellular conductivities were then added to give a total percentage relative error and the same was done for the intracellular conductivities except that fibre rotation errors were also included. These total percentage relative errors, for 
Table 3: Total percentage relative errors for extracellular conductivities (top half of the table) and for intracellular conductivities plus fibre rotation (bottom half of the table), found when retrieving the parameters from the indicated studies, using either a single pass (first column of each pair) or the usual two-pass method of Section 3 (second column of each pair).

\begin{tabular}{ccccc}
\hline Noise & $\begin{array}{c}\text { Hooks Total } g_{e} \\
\text { single }\end{array}$ & $\begin{array}{c}\text { MacLachlan Total } g_{e} \\
\text { two-pass }\end{array}$ & single & two-pass \\
\hline 1 & 0.4 & 0.3 & 0.3 & 0.7 \\
2 & 1.0 & 0.6 & 0.2 & 0.7 \\
5 & 3.6 & 2.0 & 1.2 & 0.9 \\
\hline Noise & Hooks & Total $g_{i}+\alpha$ & MacLachlan Total $g_{i}+\alpha$ \\
& single & two-pass & single & two-pass \\
\hline 1 & 4.6 & 3.9 & 2.6 & 4.1 \\
2 & 23.9 & 6.0 & 7.1 & 8.2 \\
5 & 49.6 & 19.8 & 11.7 & 22.8 \\
\hline
\end{tabular}

both datasets (Table 1), are presented in Table 3 for the single-pass method and the usual two-pass method (with ten runs in each pass).

These results show, perhaps surprisingly, that it is possible to find all six conductivities and fibre rotation using only one pass of the inversion routine and a 'widely-spaced' electrode set. In fact, for the noise values presented here, the extracellular conductivities can be retrieved quite accurately, and nearly as accurately as in the two-pass method for the dataset of MacLachlan et al. [12]. It is, however, not possible to be definitive about this, as exactly the same noise is not added to the potentials in each of the two scenarios, due to the fact that different numbers of electrodes are being used. It is also clear, though, that the intracellular conductivities and fibre rotation are not being retrieved as accurately using the single pass as in the two-pass method. The difference between the two methods also appears to be increasing with increasing noise. This leads to the conclusion that the two-pass inversion method is superior to the single-pass inversion method.

The fact that the single pass with a 'widely-spaced' electrode set is actually able to retrieve the extracellular conductivities accurately can perhaps be explained in terms of the concept of sensitivity of the inversion algorithm to changes in the conductivity values or fibre rotation. This is calculated by adding $10 \%$ error individually to each parameter and determining the potentials on the measuring array. These are compared, via relative errors, with the potentials produced by the original parameters; that is, at each electrode

$$
\text { sensitivity }(\%)=\left|\frac{\phi_{e}-\phi_{e c}}{\phi_{e}}\right| \times 100
$$


Table 4: Sensitivity (to $10 \%$ changes in given conductivity values from the indicated studies).

\begin{tabular}{crrrrrrr}
\hline & $g_{\text {el }}$ & $g_{\text {et }}$ & $g_{\text {en }}$ & $g_{i l}$ & $g_{i t}$ & $g_{i n}$ & $\alpha$ \\
\hline \multicolumn{7}{c}{ Hooks et al. [11] } \\
1st pass & 84 & 55 & 89 & 2 & 2 & 0.4 & 5 \\
2nd pass & 2795 & 494 & 3340 & 461 & 48 & 174 & 194 \\
\hline \multicolumn{7}{c}{ MacLachlan et al. [12] } \\
1st pass & 35 & 40 & 66 & 1 & 3 & 2 & 5 \\
2nd pass & 275 & 138 & 197 & 56 & 60 & 52 & 27
\end{tabular}

where $\phi_{e}$ and $\phi_{e c}$ are simulated potentials on the electrode array for the original and $10 \%$ increased conductivities, respectively.

A very interesting conclusion that can be drawn from the sensitivities in Table 4 is that first pass is very sensitive to the extracellular conductivities, but not to the intracellular values, whereas the second pass is sensitive to both, albeit still much more sensitive to the extracellular conductivities than the intracellular ones. This would explain why it is possible to retrieve the extracellular conductivities using the 'widely-spaced' source and sink electrodes of the single pass. In addition, the fibre rotation sensitivities are of the same order as the intracellular sensitivities in each pass, consistent with requiring the algorithm to find the fibre rotation in the second pass and not the first.

It is interesting to speculate as to why the inversion algorithm is sensitive to some parameters in the first pass and to others only in the second. For the conductivities this is consistent with the physical situation [31] where the 'closely-spaced' source and sink electrodes result in almost all the current entering the extracellular space, with the result that the algorithm is sensitive to only the extracellular conductivities. On the other hand, the 'widely-spaced' source and sink electrodes direct some of the current into the intracellular space as well, resulting in some sensitivity to the intracellular conductivities, as well as still being sensitive to the extracellular conductivities. The fact that the inversion algorithm is sensitive to fibre rotation only in the second pass possibly occurs for a different reason; that is, it is only when the source and sink electrodes are sufficiently far apart that there is enough tissue for the change in fibre rotation angle to be detectable. Or, looking at this from a mathematical point of view, the conductivity tensors in Equation (3) involve either combinations of $g_{i}$ and $\alpha$ values or $g_{e}$ and $\alpha$ values, so perhaps it is not possible to find $\alpha$ until the algorithm is sensitive to the $g_{i} \mathrm{~s}$, that is in the second pass. 


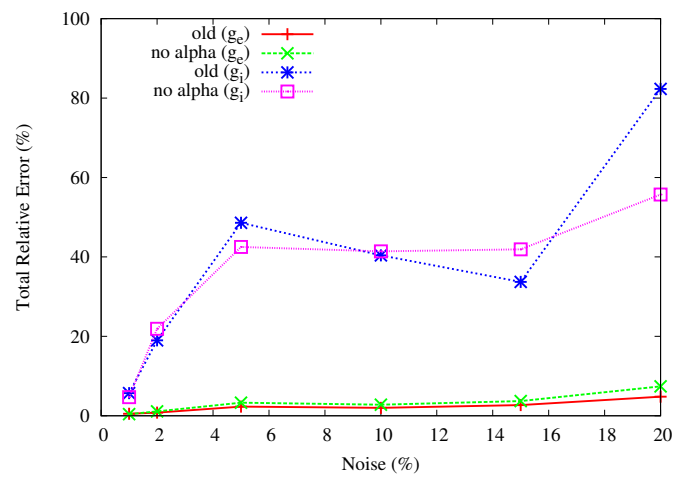

(a) Hooks [9]

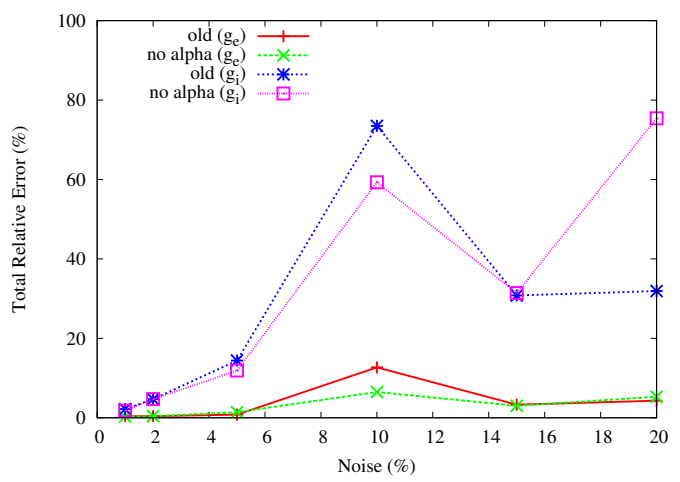

(b) MacLachlan et al. [12]

Figure 2: Total percentage relative errors, for the three extracellular conductivities (first pass) and the three intracellular conductivities (second pass), found using the usual method of Section 3 (denoted 'old') and the method with fibre rotation not retrieved (denoted 'no alpha'), for various noise levels and the datasets of (a) Hooks [9] and (b) MacLachlan et al. [12].

\subsubsection{The effect, on the inversion, of not retrieving fibre rotation}

Since there are experimental methods, such as histology [11], that can determine fibre rotation through the tissue, in this section we consider whether it is possible to retrieve the conductivities more accurately if fibre rotation is kept fixed. This means that, in the first pass, the inversion routine would retrieve six conductivities only and the second pass would refine the results for the three intracellular conductivities, as compared with the seven and four parameters retrieved in the first and second passes, respectively, of the usual method (Section 3). To achieve this, the inversion algorithm is modified by setting $\gamma_{2}=0$ in equations (11) and (12), and the same noisy potentials are used in each of the two scenarios.

The two methods are compared via total percentage relative errors (Section 4.1.1) for various noise values in Figure 2, with 'old' denoting the usual method and 'no alpha' denoting the method where fibre rotation is held constant. It seems that, for the extracellular conductivities, in most cases there is very little difference between the two methods. This result is also consistent with the sensitivity results (Table 4), which indicate that the first pass is not sensitive to the values of $\alpha$. The results for the intracellular conductivities are mixed and do not conclusively indicate whether one method is superior to the other. This may partly be due to the fact that the starting values for the second pass are different in the two methods because they come from the first pass. Given the inconclusive results, it seems that it 
would be sensible to continue to retrieve fibre rotation, in addition to the conductivities, and then use this as a check on the results by comparing it with values found by histology [11].

\subsection{Realistically Sized Simulated Datasets}

The second part of this work is a study of the data analysis that is required as part of the inversion algorithm, when large sets of potentials are considered, as would be the case under realistic experimental conditions.

In this section, two passes are used and the six conductivities $\left(g_{p q}, p=\right.$ $i, e, q=l, t, n)$ as well as fibre rotation are retrieved. For each dataset (Hooks [11] and MacLachlan et al. [12]), pass (first or second) and each noise level (5-40\%), random noise is added to one hundred sets of simulated potentials and from each of these, sets of seven parameters are retrieved using the inversion algorithm. As discussed in our earlier work [21], the inversion algorithm occasionally converges to non-physiological conductivity values. This was previously [21] dealt with by applying an exclusion criterion that rejected a set of parameters if three or more were each outside one standard deviation (std) from the mean. This work re-visits this criterion to see if it is possible to achieve more accurate conductivities using a different method. This is motivated by the fact that with 100 sets of parameters, more nonphysiological values may well be found.

\subsubsection{Data characteristics}

Firstly, the data are studied to see if they are normally distributed, since this would provide a justification for removing 'outliers' based on the std from the mean. A histogram for each of the retrieved conductivities is given in Figure 3 for $10 \%$ noise for the Hooks dataset [9] and it shows that the data are approximately normally distributed around the exact values, as given in Table 1. After some investigation, it is decided that individual conductivity values will not be excluded, but rather whole sets of parameters, since the algorithm is attempting to find a minimum in the whole parameter space.

\subsubsection{Comparison of 1 st pass inversion methods}

Three different whole-set exclusion methods are evaluated for the first pass: keep all the data ('all'); keep the set of parameters if all three extracellular conductivities are within one std of the mean ('new') and reject a set if any three parameters are outside one std of the mean ('old'). The last of these is the criterion that has been used in earlier work [21] and it differs from the 'new' criterion in that the 'old' method considers all of the parameters. 

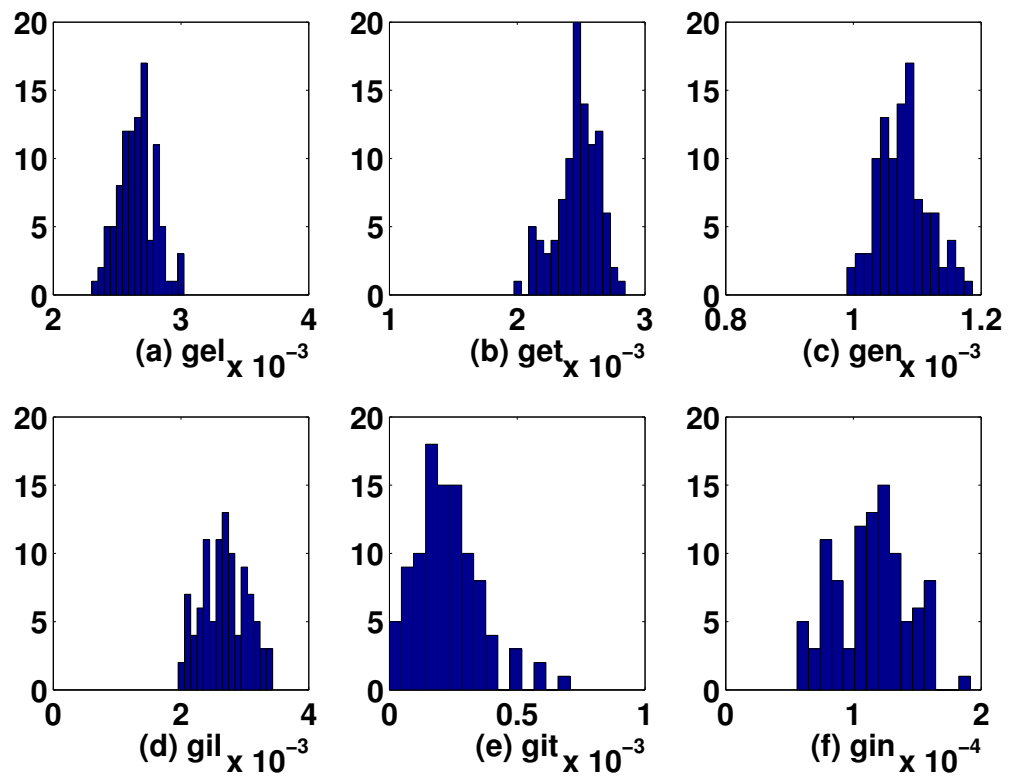

Figure 3: Histogram of number of occurrences of the indicated Hooks conductivity values [11], for 100 simulations and $10 \%$ noise, for (a) $g_{e l}$, (b) $g_{e t}$ and (c) $g_{e n}$ in the first pass and (d) $g_{i l}$, (e) $g_{i t}$ and (f) $g_{i n}$ in the second pass.

The three methods, 'all', 'new' and 'old', are compared in Figure 4 via the total relative error for the three extracellular conductivities, for each of the two starting datasets (Table 1). Using the exclusion criteria for the 'new' method results in approximately 53\% (50\% Hooks, 56\% MacLachlan) of the parameter sets being retained, whereas in the 'old' method approximately 87\% (91\% Hooks, $83 \%$ MacLachlan) are retained. It appears that, for either dataset, there is little difference in the methods for added noise up to $30 \%$, although 'new' method is slightly superior to the 'old' method in the majority of cases (excluding mid-range noise for the Hooks dataset). However, for the higher noise values of $35 \%$ and $40 \%$, it is clear that removing some of the parameter sets, as in either of the 'new' or 'old' methods, improves the retrieval accuracy.

\subsubsection{Comparison of 2nd pass inversion methods}

Similar exclusion criteria to those in the first pass are settled on for comparison in the second pass, that is, 'all', 'new' and 'old' methods. These 


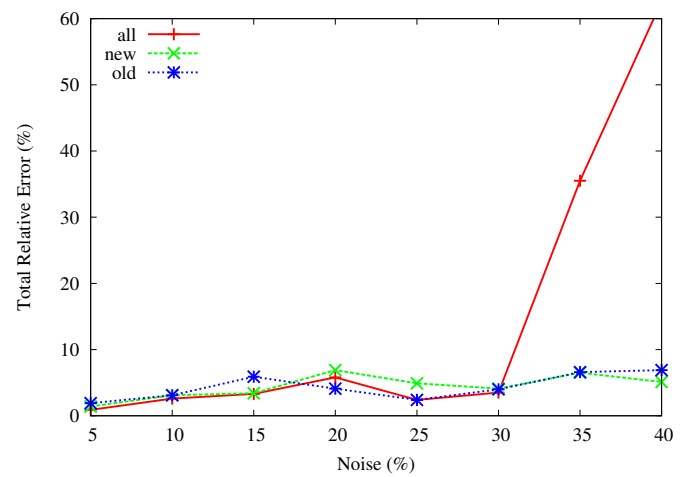

(a) Hooks [9]

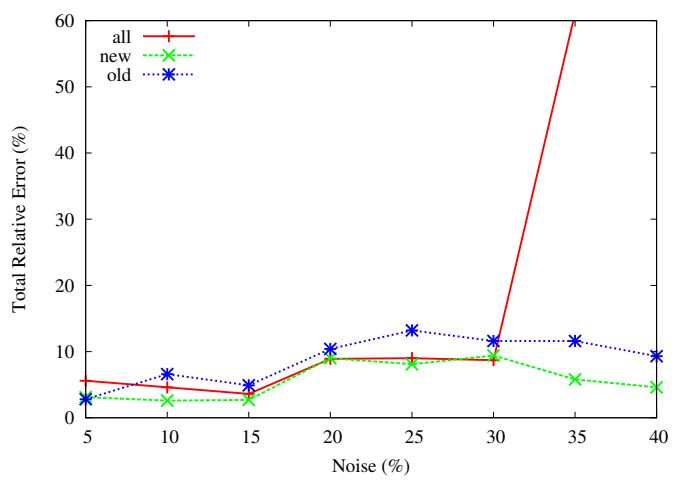

(b) MacLachlan et al. [12]

Figure 4: Total percentage relative errors found in the first pass, for the three extracellular conductivities, found using each of the three methods (see Section 4.2.2 for definitions), for various noise levels and the datasets of (a) Hooks [9] and (b) MacLachlan et al. [12].

differ from the first pass only in the number of parameters that are considered in the 'old' method, since only four parameters are retrieved in the second pass. Other methods (for example, just considering the intracellular conductivities and not fibre rotation) are initially evaluated, but since they are found to be inferior to the 'new' method, they are not presented.

In a similar fashion to the first pass, the three methods are compared for the second pass in Figure 5. In this case, only around $30 \%$ of the records are retained for the 'new' method, compared with approximately $90 \%$ for the 'old' method. Using total percentage relative errors (in this case a sum of four parameters not three as in the first pass), it can be seen (Figure 5) that the inversion method is able to retrieve the MacLachlan conductivities more accurately, in most cases, than the Hooks conductivities. In the MacLachlan case, the accuracy of all three methods is similar, although the 'old' method appears slightly superior for the highest noise value. This is not the case, however, for the Hooks conductivities, where the 'new' method is generally superior, especially for the two highest noise values. In the light of these results, it would seem prudent to use the 'new' method, since it is of similar accuracy to the other methods in the MacLachlan case and is clearly superior for high noise values in the case of the Hooks dataset.

\subsection{Accuracy of parameter retrievals}

In this section, the accuracy of the inversion algorithm will be discussed in terms of the mean percentage relative errors found using the 'new' method. For the first pass, these results are given in Supplementary Table 


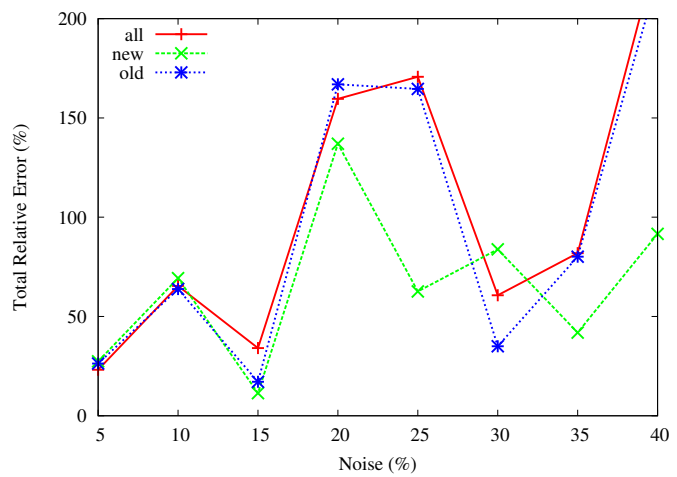

(a) Hooks [9]

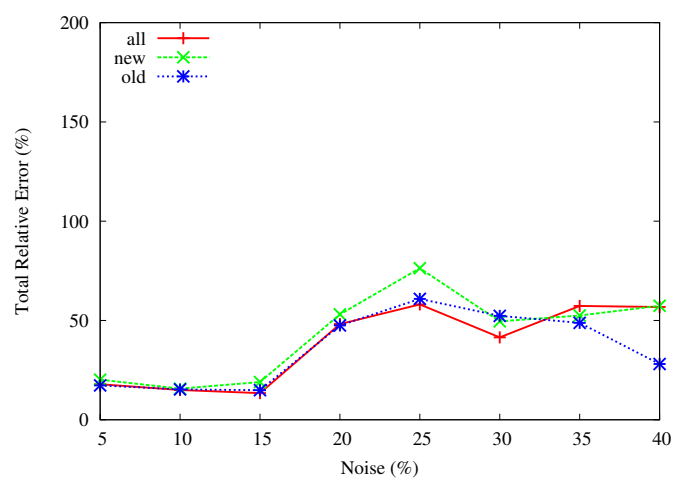

(b) MacLachlan et al. [12]

Figure 5: Total percentage relative errors found in the second pass, for the three intracellular conductivities plus fibre rotation, found using each of the three methods (see Section 4.2.3 for definitions), for various noise levels and the datasets of (a) Hooks [9] and (b) MacLachlan et al. [12].

1 and Figure 4, bearing in mind that the errors plotted in Figure 4 are the sum of the errors for the three values. It is found that the inversion algorithm is able to retrieve all three extracellular conductivities extremely accurately from realistically large sets of (simulated) experimental measurements of heart potentials, generated from either of the two initial conductivity datasets (Table 1). The maximum relative error for these values is around $5 \%$ and averaging over all the extracellular mean percentage relative errors for the 'new' method in Supplementary Table 1 gives a relative error of $1.7 \%$ on average, despite the added noise values being as high as $40 \%$. In fact, the total extracellular error (Figure 4) does not appear to increase once the added noise reaches $20 \%$.

The situation is rather different for the three intracellular conductivities and fibre rotation (Supplementary Table 2 and Figure 5). For example, the total errors are often considerably less for the MacLachlan dataset than for Hooks (although not for 15\%, 25\% and 35\% noise). However, for both datasets there are two parameters, intracellular longitudinal conductivity $g_{i l}$ and fibre rotation $\alpha$, that are still retrieved quite accurately, with percentage relative errors that are generally around the level of the added noise. In each of these cases, the average of all the 'new' method percentage relative errors in Supplementary Table 2 is less than $8 \%$. Clearly, the intracellular transverse and normal conductivities cannot be retrieved as accurately as this and there are also differences in the accuracy of the algorithm for the two datasets. In fact, for the MacLachlan dataset the averages of all the mean 
percentage relative errors in Supplementary Table 2 for the 'new' method are $13.5 \%$ and $13 \%$ ( $g_{i t}$ and $g_{i n}$, respectively), which is still reasonably accurate. This is not the case for the Hooks dataset where the accuracy is poor for a number of cases. However, even in the Hooks case, in all but four of the cases (which generally correspond to lower values of added noise), the percentage relative errors for $g_{i t}$ and $g_{i n}$ are less than twice the added noise.

\section{Conclusions}

The purpose of this work was to investigate the degree of accuracy that could be achieved when retrieving six cardiac bidomain conductivity values, $g_{e l}, g_{e t}, g_{e n}, g_{i l}, g_{i t}, g_{i n}$, and fibre rotation angle $\alpha$, from realistically large datasets of heart potential measurements. Initially, two aspects of the inversion algorithm were examined to see if more accurate retrievals could be achieved.

The first found that, while it is possible to retrieve all six bidomain conductivities and fibre rotation with a single pass of the algorithm using a 'widely-spaced' set of electrodes, the accuracy drops off as noise increases and so this method is not recommended. The fact that this is possible at all can be explained in terms of the concept of sensitivity, in that the second pass is still very sensitive to the extracellular conductivities.

The second investigation considered whether not retrieving the fibre rotation (since it can be found by other means, such as histology) would improve the accuracy of the algorithm. It was found that not retrieving fibre rotation in the first pass had almost no effect on the results. This is consistent with the fact that the first pass is not sensitive to the values of fibre rotation. It was also concluded that the effect of not retrieving fibre rotation in the second pass was not sufficiently large to make it worthwhile. So it is suggested that the algorithm should still continue to retrieve fibre rotation and the result should then be compared with the value found in histology studies to provide a check on the results.

The main aspect of this work was an in silico study using large (100) sets of potential measurements from which sets of seven parameters (six conductivities and fibre rotation) were retrieved. Several data analysis methods, for removing non-physiological parameters, were considered and the best overall method was identified. This data analysis method (denoted 'new') was combined with the usual two-pass inversion technique to retrieve the six conductivities and fibre rotation, for the datasets of Hooks [11] and MacLachlan et al. [12] and for a range of added noise values. It was found 
that for added noise of up to $40 \%$ the three extracellular conductivity values can be retrieved with mean relative errors of less than $2 \%$ on average. Two of the other parameters (the intracellular conductivity and fibre rotation) can also be retrieved very accurately with mean relative errors of less than $8 \%$ on average. Although the remaining intracellular conductivity values (transverse and normal) are more difficult to retrieve, it is generally the case that their mean relative errors are less than twice the noise added, particularly for higher values of noise.

Plans for future work include construction of the multi-electrode array and making potential measurements in vivo in an animal model. The techniques considered in this work will then be used to retrieve the six bidomain conductivity values as well as fibre rotation.

\section{References}

[1] Henriquez, C. S. A brief history of tissue models for cardiac electrophysiology IEEE Transactions in Biomedical Engineering, 61(5):1457-1465, 2014.

[2] Smaill, B. H., Zhao, J., and Trew, M. L. Three-dimensional impulse propagation in myocardium: Arrhythmogenic mechanisms at the tissue level Circulation Research, 112:831-833, 2013.

[3] Clayton, R. H., Bernus, O., Cherry, E. M., Dierckx, H., Fenton, F. H., Mirabella, L., Panfilov, A. V., Sachse, F. B., Seemann, G., and Zhang, H. Models of cardiac tissue electrophysiology: Progress, challenges and open questions Progress in Biophysics and Molecular Biology, 104(13):22-48, 2011.

[4] Clerc, L. Directional differences of impulse spread in trabecular muscle from mammalian heart Journal of Physiology, 255:335-346, 1976.

[5] Roberts, D. E., Hersh, L. T., and Scher, A. M. Influence of cardiac fiber orientation on wavefront voltage, conduction velocity and tissue resistivity in the dog Circ. Res., 44:701-712, 1979.

[6] Roberts, D. E. and Scher, A. M. Effects of tissue anisotropy on extracellular potential fields in canine myocardium in situ Circ. Res., 50:342-351, 1982.

[7] Roth, B. J. Electrical conductivity values used with the bidomain model of cardiac tissue IEEE Transactions on Biomedical Engineering, 44(4):326-328, April 1997. 
[8] Johnston, P. R. and Kilpatrick, D. The effect of conductivity values on ST segment shift in subendocardial ischaemia IEEE Transactions on Biomedical Engineering, 50(2):150-158, February 2003.

[9] Hooks, D. A., Trew, M. L., Caldwell, B. J., Sands, G. B., LeGrice, I. J., and Smaill, B. H. Laminar arrangement of ventricular myocytes influences electrical behavior of the heart Circulation Research, 101(10):e103-112-e103-112, 112007.

[10] Hooks, D. and Trew, M. Construction and validation of a plunge electrode array for three-dimensional determination of conductivity in the heart IEEE Transactions on Biomedical Engineering, 55(2):626-635, 2008 .

[11] Hooks, D. Myocardial segment-specific model generation for simulating the electrical action of the heart BioMedical Engineering OnLine, 6(1):21-21, 2007.

[12] MacLachlan, M. C., Sundnes, J., and Lines, G. T. Simulation of ST segment changes during subendocardial ischemia using a realistic 3D cardiac geometry IEEE Transactions on Biomedical Engineering, 52(5):799-807, 2005.

[13] Johnston, P. R. Cardiac conductivity values - a challenge for experimentalists? Noninvasive Functional Source Imaging of the Brain and Heart 82011 8th International Conference on Bioelectromagnetism (NFSI \& ICBEM), pages 39-43, 13-16 May 2011.

[14] Caldwell, B. J., Trew, M. L., Sands, G. B., Hooks, D. A., LeGrice, I. J., and Smaill, B. H. Three distinct directions of intramural activation reveal nonuniform side-to-side electrical coupling of ventricular myocytes Circulation: Arrhythmia and Electropysiology, 2:433-440, 2009.

[15] Pollard, A. E., Ellis, C. D., and Smith, W. M. Linear electrode arrays for stimulation and recording within cardiac tissue space constants Biomedical Engineering, IEEE Transactions on, 55(4):1408-1414, 2008.

[16] Pollard, A. E. and Barr, R. C. A biophysical model for cardiac microimpedance measurements American Journal of Physiology-Heart and Circulatory Physiology, 298:H1699-H1709, 2010.

[17] Graham, L. S. and Kilpatrick, D. Estimation of the Bidomain conductivity parameters of cardiac tissue from extracellular potential distribu- 
tions initiated by point stimulation Annals of Biomedical Engineering, 38(12):3630-3648, 2010.

[18] Eliad Gilboa, P. S. L. R. and Nehorai, A. Estimating electrical conductivity tensors of biological tissues using microelectrode arrays Annals of Biomedical Engineering, 40(10):2140-2155, 2012.

[19] Xi, J., Lamata, P., Lee, J., Moireau, P., Chapelle, D., and Smith, N. Myocardial transversely isotropic material parameter estimation from in-silico measurements based on a reduced-order Kalman filter Journal of the Mechanical Behaviour of Biomedical Materials, 4:10901102, 2011.

[20] Johnston, B. M. and Johnston, P. R. A multi-electrode array and inversion technique for retrieving six conductivities from heart potential measurements Medical and Biological Engineering and Computing, 51(12):1295-1303, 2013.

[21] Johnston, B. M. Using a sensitivity study to facilitate the design of a multi-electrode array to measure six cardiac conductivity values Mathematical Biosciences, 244:40-46, 2013.

[22] Hooks, D. A., Tomlinson, K. A., Marsden, S. G., LeGrice, I. J., Smaill, B. H., Pullan, A. J., and Hunter, P. J. Cardiac microstructure: Implications for electrical propagation and defibrillation in the heart Circulation Research, 91(4):331-338, 82002.

[23] Johnston, B. M. and Barnes, J. P. Exploiting GPUs to investigate an inversion method that retrieves cardiac conductivities from potential measurements ANZIAM Journal, 55:C17-C31, March 2014.

[24] Schmitt, O. H. Biological information processing using the concept of interpenetrating domains In Leibovic, K. N., editor, Information Processing in the Nervous System, chapter 18, pages 325-331 SpringerVerlag, New York, 1969.

[25] Tung, L. A Bi-domain model for describing ischaemic myocardial D$C$ potentials $\mathrm{PhD}$ thesis, Massachusetts Institute of Technology, June 1978.

[26] Geselowitz, D. B. and Thorsson, H. T. Multiple dipole inverse solutions from human body surface electrocardiograms In Computers in Cardiology, pages 461-464 IEEE Computer Society, 1983. 
[27] Johnston, P. R., Kilpatrick, D., and Li, C. Y. The importance of anisotropy in modelling ST segment shift in subendocardial ischaemia IEEE Transactions on Biomedical Engineering, 48(12):1366-1376, December 2001.

[28] Johnston, B. M., Johnston, P. R., and Kilpatrick, D. A new approach to the determination of cardiac potential distributions: Application to the analysis of electrode configurations Mathematical Biosciences, 202(2):288-309, 2006.

[29] Johnston, B. M. and Johnston, P. R. Possible four-electrode configurations for measuring cardiac tissue fibre rotation IEEE Transactions on Biomedical Engineering, 54(3):547-550, 2007.

[30] Johnston, B. M., Johnston, P. R., and Kilpatrick, D. Analysis of electrode configurations for measuring cardiac tissue conductivities and fibre rotation Annals of Biomedical Engineering, 34(6):986-996, June 2006.

[31] Plonsey, R. and Barr, R. C. The four-electrode resistivity technique as applied to cardiac muscle IEEE Transactions on Biomedical Engineering, 29(7):541-546, 1982.

[32] Weidmann, S. Electrical constants of trabecular muscle from mammalian heart Journal of Physiology, 210:1041-1054, 1970.

[33] R. C. Barr and R. Plonsey Electrode systems for measuring cardiac impedances using optical transmembrane potential sensors and interstitial electrodes - Theoretical design IEEE Transactions on Biomedical Engineering, 50(8):925-934, 2003.

[34] Rush, S., Abildskov, J. A., and McFee, R. Resistivity of body tissues at low frequencies Circulation Research, 12:40-50, 1963.

[35] Aster, R. C., Borchers, B., and Thurber, C. H. Parameter Estimation and Inverse Problems Elsevier Academic Press, Burlington, 2005.

[36] Kuntsevich, A. and Kappel, F. SolvOpt: The solver for Local Nonlinear Optimisation Problems, version 1.1 in $C$ Institute for Mathematics: Karl-Franzens University of Graz, 1997.

[37] Shor, N. Z. Minimization methods for Non-Differentiable Functions, volume 3 of Springer Series in Computational Mathematics SpringerVerlag, 1985. 